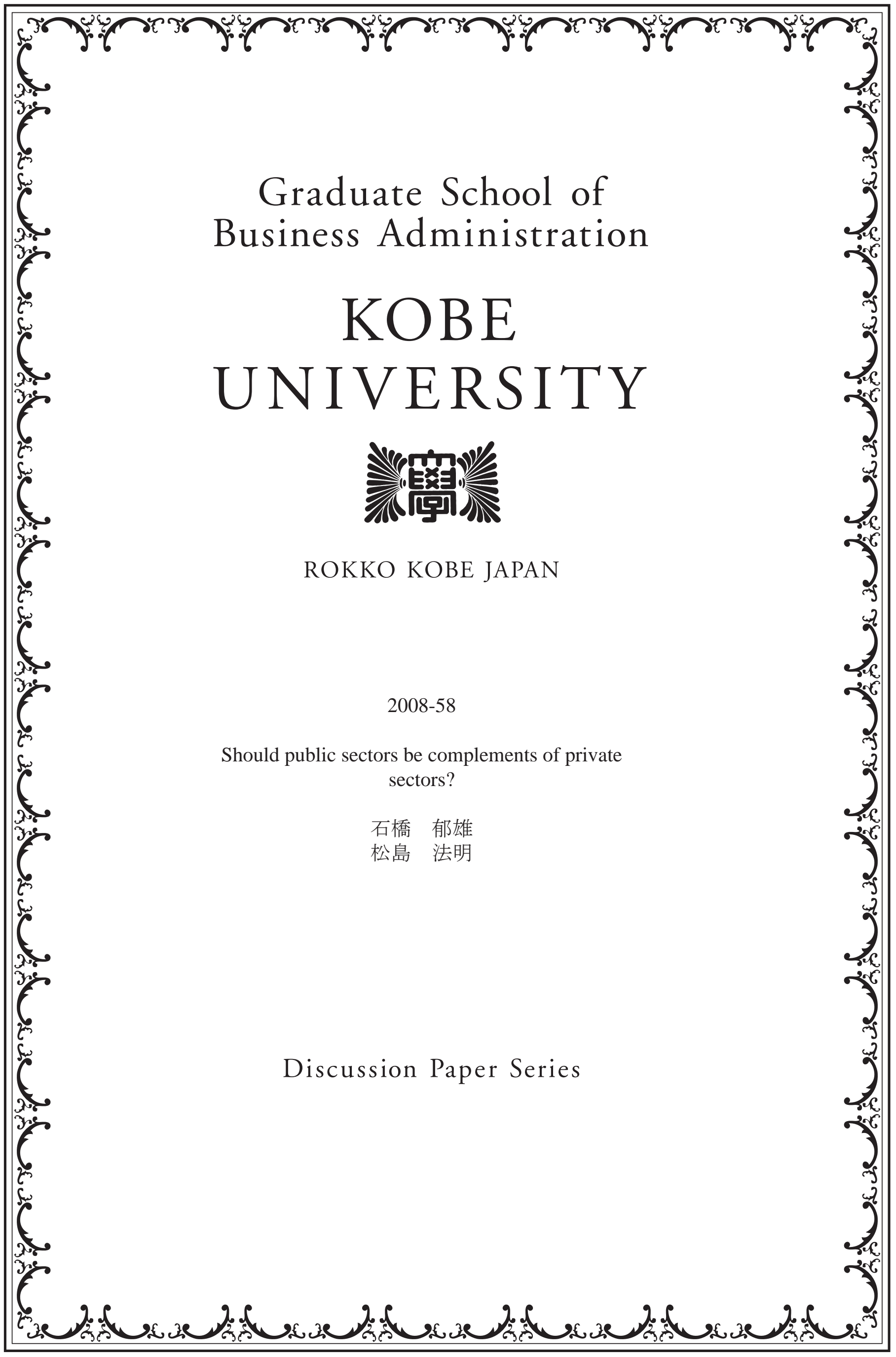




\title{
Should public sectors be complements of private sectors?*
}

\author{
Ikuo Ishibashi ${ }^{\dagger}$ \\ Department of Economics, Aoyama Gakuin University \\ Noriaki Matsushima ${ }^{\ddagger}$ \\ Graduate School of Business Administration, Kobe University
}

December 1, 2008

\begin{abstract}
This paper discusses competition between high-quality private service providers that maximize their own profits and a low-quality public service provider that maximizes social surplus. There are two heterogeneous consumer groups: those who demand only high-quality services and those who care little whether services are highor low-quality. The setting reflects the fact that some consumers feel dissatisfaction with public service providers. We show that, under certain conditions, social welfare is smaller when there is a public service provider than when there is not. The result holds even though the efficiency of the public service is equal to that of the private services.
\end{abstract}

JEL classification: H42, L13, I12

Key words: public versus private sectors, quality, differentiation, mixed markets.

${ }^{*}$ We would like to thank Hideki Konishi, Naoko Nishimura, Fumihiko Numao, Debashis Pal, Ryusuke Shinohara, Ryuichi Tanaka, and seminar participants at Cincinnati University, Osaka University, Shinshu University, and Tokyo Institute of Technology for their helpful comments. The authors gratefully acknowledge financial supports from Grant-in-Aid for Encouragement of Young Scientists from the Japanese Ministry of Education, Science and Culture. Needless to say, we are responsible for any remaining errors.

${ }^{\dagger}$ Ikuo Ishibashi: Faculty of Economics, Aoyama Gakuin University 4-4-25 Shibuya, Shibuya-ku, Tokyo 150-8366 Japan. E-mail: ishibash@econ.aoyama.ac.jp

$¥$ Noriaki Matsushima (Correspondence author): Graduate School of Business Administration, Kobe University, 2-1 Rokkodai, Nada, Kobe, Hyogo 657-8501, Japan. Phone: +81-78-803-6981. Fax: +81-78803-6977. E-mail: nmatsush@kobe-u.ac.jp 


\section{Introduction}

In vertically differentiated mixed markets, ${ }^{1}$ it is often observed that public firms provide lower-quality products at a low price. For example, in Japan, public nurseries provide fewer convenient services concerning opening hours than private ones. In 2006, although more than $80 \%$ of private nurseries were open more than 11 hours, fewer than $50 \%$ of the public ones were open more than 11 hours (research on facilities for social welfare 2006; Ministry of Health, Labor, and Welfare). Public nursery services are insufficient for some parents because of constraints concerning their working hours. ${ }^{2}$ In reality, although public nurseries accept children less than $95 \%$ of their capacity, private ones accept children more than $110 \%$ of their capacity even though the private nurseries are more expensive that the public ones. These facts suggest that the services provided by the public sector are less convenient. Another example is the existence of public and private sport facilities (e.g., gyms and tennis courts). Public sports facilities are frequently poorly equipped; however, they generally charge lower fees than their private counterparts. ${ }^{3}$ Health care systems are another example in which public service providers are cheaper and of poorer quality. ${ }^{4}$ In

\footnotetext{
${ }^{1}$ A market is called mixed when profit-maximizing private firms and a welfare-maximizing public firm coexist. Mixed markets are widely observed in Europe, Canada, and Japan. One of the most important questions in studies of mixed markets is whether or not a public firm should be privatized. Because the public firm's aggressive behaviors often cause private firms' undesirable reactions through market, the answer to the question is not obvious even if public firms are properly operated without efficiency problems such as moral hazard. See Bös $(1986,1991)$ and Nett (1993) for excellent surveys of mixed oligopolies. Recently, the literature on mixed oligopoly has become richer and more diverse. For example, Poyago-Theotoky (1998), Corneo and Rob (2003), Ma (2003), Matsumura and Matsushima (2004), and Ishibashi and Matsumura (2006) investigate cost differences between public and private sectors. Pal (1998), Matsumura (2003), and Lu (2006) discuss the endogenous role. Cremer et al. (1991), Matsushima and Matsumura (2003), Li (2006), and Lu and Poddar (2007) analyze endogenous product differentiation.

${ }^{2}$ We do not want to suggest that the staff of public nurseries are inferior to those in private ones. The emphasis is on the differences in the hours of service provided.

${ }^{3}$ Glazer and Niskanen (1997) also mention that governmental facilities are often small and of poor quality and that many services provided by such facilities are also provided privately, for example, as in public versus private schools and universities, public versus private medical care, and public mass transit versus the use of privately owned automobiles.

${ }^{4}$ Several researchers investigate the interaction between lower-quality health care service by public sector and higher-quality health care service by private sector. The main concerns of them are summarized as follows: quality and regulation (Barros and Martinez-Giralt (2002)), waiting list (Barros and Olivella
} 
many developed and developing countries, service providers in traditional public health systems have typically faced very weak incentives to respond to patient preferences and their perceptions of quality. One indication of the resulting quality deficiencies is the large number of patients who choose to seek care in the private sector, despite the availability of public sector services provided at no cost. ${ }^{5}$ For instance, when someone has a cold, he/she has several choices for seeking treatment. First, the patient could go to a (private/public) hospital; second, the patient could buy and consume cold medicine; and third, the patient could rest and sleep. If the patient wanted more thorough treatment, he could go to a private hospital. If the patient did not feel that more care was needed, he could go to a public hospital, take cold medicine, or sleep more.

One rationale for the existence of public firms is that they are socially desirable, even though they are unprofitable. For example, when only a few consumers show concern for quality, no private firm would offer lower-quality services for less. ${ }^{6}$

Although public firms seem to be socially desirable, we demonstrate in this paper that, in some situations, the establishment of public firms diminishes social welfare.

In more precise terms, we consider the following situation based on the work of Ishibashi and Matsushima (2008). There are two heterogeneous consumer groups. One consists of consumers who demand high-quality products. Low-quality products are worth little to them (we call them quality conscious). ${ }^{7}$ The other consists of consumers who care (2005), March and Schroyen (2005)), and physician dual practice (Rickman and McGuire (1999), Gonzàlez (2004), Biglaiser and Ma (2007), Brekke and Sørgard. (2007)). García-Prado and González (2007) provide an excellent survey in the topic of physician dual practice. Those papers do not consider mixed oligopoly models with those heterogeneous consumer groups discussed here.

${ }^{5}$ The phenomenon is called as bypassing health provider in the literature of health economics. After Akin and Hutchinson (1999) show it as an important phenomenon in Sri Lanka, several researchers investigate this kind of problems in some developing countries (see Leonard et al. (2002) and Gauthier and Wane (2008)).

${ }^{6}$ If there is an entry barrier, it is also possible that no private firm wants to produce lower-quality products even if it can gain a positive profit. For example, when competition in the large high-end market is more profitable than monopoly in the small low-end market, no private firms may enter the small low-end market.

7 Alternatively, it is assumed that consumers in this group have sufficiently larger willingness to pay for high-quality products than that for low-quality products. 
little whether products are high- or low-quality. Therefore, they buy products with the lowest price (we call them price conscious). High-quality (resp. low-quality) products are produced by private firms (resp. a public firm if it is established). In this setting, we show that the establishment of a public firm worsens social welfare if the size of the former (quality conscious) consumer group is medium.

Ironically, the driving force of this result is the public firm's aggressive behavior in the lower-quality market. The welfare-maximizing public firm behaves aggressively in the lower-quality market and the resulting price of lower-quality products falls substantially. This causes private firms to focus on quality-conscious consumers because no profit-maximizing private firm has an incentive to sell its higher-quality products to priceconscious consumers in this situation. As a result, the price of higher-quality products is kept relatively high, and the welfare loss for quality-conscious consumers who give up buying higher-quality products becomes large. If the relative size of price-conscious consumers is small in comparison to that that of quality-conscious consumers, this negative effect outweighs the positive effect of the low price set by the public firm for price-conscious consumers.

We also note that, unlike many existing studies with standard results, we investigate not only whether or not privatization is preferable from the viewpoint of social welfare but also whether or not the nonexistence (or exit) of a public firm is preferable. We believe that these comparisons are important to learn that the establishment of public firms can be harmful to social welfare.

In this paper, a theoretical contribution is made to studies of mixed markets. The technological assumptions required for our result (the nonexistence (or exit) of a public firm is preferable) are different from those in studies of mixed markets, such as quadratic production cost (De Fraja and Delbono (1989)), variety expansion with free entry (Anderson et al. (1997)), excess R\&D investments (Matsumura and Matsushima (2004)), and product positioning (Matsushima and Matsumura (2006)). Since our paper does not include those properties, we provide a new theoretical insight on the topic of mixed markets. 
The remainder of this paper is organized as follows. Section 2 presents the basic model. Section 3 presents the main result. Section 4 concludes the paper.

\section{Model}

We consider an industry with two differentiated products $(h$ and $l$ ). For convenience, we call $h$ and $l$ high-quality and low-quality products, respectively. There are $n$ private firms that produce $h$ at a constant marginal cost normalized to zero and a public firm (firm $0)$ that produces $l$ at a constant marginal cost normalized to zero. ${ }^{8}$ Each private firm maximizes its own profit, and the public firm maximizes social welfare. ${ }^{9}$ No fixed cost is assumed for production. Let $q_{i}$ be firm $i$ 's output level $(i=0,1, \ldots, n)$. In addition, define $q \equiv\left(q_{1}, \ldots, q_{n}\right)$.

We assume that there are two groups of consumers, $H$ (the high-end market) and $L$ (the low-end market). For simplicity, we consider a polar case of the heterogeneity of consumer groups. The consumers in $H$ demand only $h$; that is, the quality of $l$ is not at all sufficient for the consumers in $H$ (see Figure 1).

\section{[Figure 1 here]}

Let $p^{h}$ be the price of $h$. The demand function of this high-end market, $D^{H}\left(p^{h}\right)$, is given by ${ }^{10}$

$$
D^{H}\left(p^{h}\right)= \begin{cases}0 & \text { if } p^{h} \in(1, \infty) \\ 1-p^{h} & \text { if } p^{h} \in[0,1]\end{cases}
$$

\footnotetext{
${ }^{8}$ Even if there are private firms that produce $l$, our main results still hold. As we will see later, a public firm with a constant marginal cost (normalized to zero) supplies $l$ so that the price of $l$ becomes zero whether or not there are private firms that produce $l$.

${ }^{9}$ In this paper, the government is not permitted to nationalize more than one firm. As pointed out by Merrill and Schneider (1966), the most efficient outcome is achieved by the nationalization of all firms, if nationalization does not change the costs of firms (i.e., no X-inefficiency in the public firm exists). The need for the analysis of a mixed oligopoly lies in the fact that it is impossible or undesirable, for political or economic reasons, to nationalize an entire sector. For example, without competitors, public firms may lose the incentive to improve their costs, resulting in a loss of social welfare. Thus, we do not consider the possibility of nationalizing all firms.

10 This demand function is derived by assuming that a typical consumer in $H$ has the willingness to pay $x$ for product $h, x$ is distributed uniformly on $[0,1]$, and the total population is 1 .
} 
The consumers in $L$ are indifferent between $h$ and $l$. In other words, the high quality of $h$ (compared with $l$ ) is of no value to consumers in $L$. Let $p^{l}$ be the price of $l$. The demand function of this low-end market, $D^{L}\left(p^{l}\right)$, is given by ${ }^{11}$

$$
D^{L}\left(p^{l}\right)= \begin{cases}0 & \text { if } p^{l} \in(a, \infty) \\ b\left(1-p^{l} / a\right) & \text { if } p^{l} \in[0, a]\end{cases}
$$

We assume $0<a \leq 1$. Note that $D^{L}\left(p^{l}\right)$ is a linear demand function such that the highest willingness to pay is given by $a$ and the largest demand (at $p^{l}=0$ ) is given by $b$. Thus, $(a, b)$ measures the relative market properties of the low-end market taking the high-end market as a reference point. ${ }^{12}$

We describe how $p^{h}$ and $p^{l}$ are determined given the above consumers. As long as $1-\sum_{i=1}^{n} q_{i} \geq a\left(1-q_{0} / b\right)$, the high-end and low-end markets are separated. In other words, no consumer in $L$ buys $h$. Therefore, $p^{h}$ is given by $1-\sum_{i=1}^{n} q_{i}$, and $p^{l}$ is given by $a\left(1-q_{0} / b\right)$. If $1-\sum_{i=1}^{n} q_{i}<a\left(1-q_{0} / b\right)$, the markets are connected. That is, some consumers in $L$ buy $h$. Because $h$ and $l$ are completely indifferent to consumers in $L$, $p^{h}=p^{l}=a\left(1+b-\left(\sum_{i=1}^{n} q_{i}+q_{0}\right)\right) /(a+b)$.

In summary, the prices are determined as follows.

$$
\begin{aligned}
& p^{h}\left(q, q_{0}\right)= \begin{cases}1-\sum_{i=1}^{n} q_{i} & \text { if } \sum_{i=1}^{n} q_{i} \leq 1-a+\frac{a q_{0}}{b} \\
\frac{a\left(1+b-\left(\sum_{i=1}^{n} q_{i}+q_{0}\right)\right)}{a+b} & \text { otherwise. }\end{cases} \\
& p^{l}\left(q, q_{0}\right)= \begin{cases}a\left(1-\frac{q_{0}}{b}\right) & \text { if } \sum_{i=1}^{n} q_{i} \leq 1-a+\frac{a q_{0}}{b} \\
\frac{a\left(1+b-\left(\sum_{i=1}^{n} q_{i}+q_{0}\right)\right)}{a+b} & \text { otherwise. }\end{cases}
\end{aligned}
$$

\footnotetext{
11 This demand function is derived by assuming that a typical consumer in $L$ has the same willingness to pay $x$ for product $h$ or for product $l, x$ is distributed uniformly on $[0, a]$, and the total population is $b$.

12 Although $a \leq 1$ is assumed for simplicity, it seems reasonable to assume that quality-conscious consumers tend to evaluate high-quality products at least as high as quality-unconscious consumers. As for $b$, no upper bound is assumed. Therefore, our analysis can cover various demand structures on the relationship between the high-end and the low-end markets.
} 
Let $\pi_{i}\left(q, q_{0}\right)$ is firm $i$ 's profit function. For $i=1,2, \ldots, n$, it can be expressed as follows.

$$
\pi_{i}\left(q, q_{0}\right)= \begin{cases}\left(1-\sum_{i=1}^{n} q_{i}\right) q_{i} & \text { if } \sum_{i=1}^{n} q_{i} \leq 1-a+\frac{a q_{0}}{b} \\ \frac{a\left(1+b-\left(\sum_{i=1}^{n} q_{i}+q_{0}\right)\right)}{a+b} q_{i} & \text { otherwise. }\end{cases}
$$

Social welfare, which is also the objective function of the public firm, can be expressed as:

$$
S W\left(q, q_{0}\right)=\left\{\begin{array}{r}
\int_{0}^{\sum_{i=1}^{n} q_{i}}(1-x) d x+\int_{0}^{q_{0}} a\left(1-\frac{x}{b}\right) d x \\
\text { if } \sum_{i=1}^{n} q_{i} \leq 1-a+\frac{a q_{0}}{b}, \\
\int_{0}^{1-a}(1-x) d x+\int_{1-a}^{\sum_{i=1}^{n} q_{i}+q_{0}} \frac{a(1+b-x)}{a+b} d x \\
\text { otherwise. }
\end{array}\right.
$$

\section{Result}

In the following subsections, we consider two polar cases: (1) a public firm produces product $l$; and (2) no public firm exists. Comparing the two cases, we derive the main result of this study.

\subsection{Case I: A public firm in the low-end market}

In this subsection, we assume that there is a public firm that maximizes social welfare in the low-end market.

To show that no private firm wants to sell its product to the consumers in $L$, we first consider the best response of the public firm. From (4), we can easily derive the best response of the public firm as follows:

$$
q_{0}(q)= \begin{cases}b & \text { if } \sum_{i=1}^{n} q_{i} \leq 1 \\ 1+b-\sum_{i=1}^{n} q_{i} & \text { otherwise }\end{cases}
$$

From (3), we can also derive the best response of each private firm as follows:

$$
q_{i}\left(q_{0}, q_{-i}\right)= \begin{cases}\frac{1-\sum_{j \neq i} q_{j}}{2}, & \text { if } \sum_{i=1}^{n} q_{i} \leq 1-a+\frac{a q_{0}}{b}, \\ \frac{1+b-\sum_{j \neq i} q_{j}-q_{0}}{2} & \text { otherwise. }\end{cases}
$$


From the equations, we have the following unique Nash equilibrium:

$$
\left(q_{0}^{*}, q^{*}\right)=\left(b, q^{x}\right) \text {, where } q^{x} \equiv(1 /(n+1), \ldots, 1 /(n+1)) .
$$

Each private firm's equilibrium profit is $1 /(n+1)^{2}$. When the public firm exists, the demand for each private firm is similar to that in which the consumers in $L$ do not exist because the public firm fully covers the demand of the consumers in $L$ to maximize social welfare. As a result, there is no room for the private firms to supply for the consumers in $L$. We summarize this result as follows.

Lemma 1 If there is a public firm, the unique Cournot-Nash equilibrium is $q_{0}=b, q^{x}=$ $(1 /(n+1), \ldots, 1 /(n+1))$. The profit of each firm and social welfare are:

$$
\pi_{I} \equiv \frac{1}{(n+1)^{2}}, \quad S W_{I} \equiv \frac{a b+(1+a b) n(n+2)}{2(n+1)^{2}} .
$$

\subsection{Case II: No public firm in the low-end market}

In this subsection, we consider a case in which the public firm exits from the low-end market and $n$ private firms can potentially sell to both groups of consumers. This case becomes a simple Cournot oligopoly game with a kinked demand curve.

There are two candidates of Cournot equilibria:

$$
q^{x} \text { and } q^{y} \equiv((1+b) /(n+1), \ldots,(1+b) /(n+1)) .
$$

This is caused by our kinked inverse demand function. $q^{x}$ (resp. $\left.q^{y}\right)$ is the Cournot equilibrium when the whole inverse demand function is given by $p^{x}(q)=1-\sum_{i=1} q_{i}$ $\left(\right.$ resp. $\left.p^{y}(q)=a\left(1+b-\sum_{i=1} q_{i}\right) /(a+b)\right)$ (see (1)). Therefore, we must check not only the local optimality (i.e., first-order condition) but also the global optimality (i.e., deviations "beyond" the kinked point). After several calculations, we obtain the following lemmas (the calculations are described in the Appendix):

Lemma $2 q^{y}=((1+b) /(n+1), \ldots,(1+b) /(n+1))$ becomes a Cournot equilibrium if

$$
a \geq \frac{(2-(n-1) b)^{2}}{(n+1)(4-(n-3) b)} \text { or } \quad b \geq \frac{2}{n-1} .
$$


The profit of each firm and social welfare are:

$$
\pi_{I I y} \equiv \frac{a(1+b)^{2}}{(n+1)^{2}(a+b)}, \quad S W_{I I y} \equiv \frac{(1-a)^{2} b+(a+b)(1+a b) n(n+2)}{2(a+b)(n+1)^{2}} .
$$

Lemma $3 q^{x}=(1 /(n+1), \ldots, 1 /(n+1))$ becomes a Cournot equilibrium if

$$
a \leq \frac{4}{(n+1)(4+(n+1) b)} \text {. }
$$

The profit of each firm and social welfare are:

$$
\pi_{I I x} \equiv \frac{1}{(n+1)^{2}}, \quad S W_{I I x} \equiv \frac{n^{2}}{2(n+1)^{2}} .
$$

Lemma 2 says that $q^{y}$ becomes an equilibrium if the low-end market is sufficiently profitable (i.e., $a$ is relatively large for a given $b$ ) or large (i.e., $b$ is higher than $2 /(n-1)$ ). The first condition is relatively easy to understand. Firms sell to the consumers in $L$ because the increase in sales outweighs the decrease in price. What the second condition implies is slightly more complicated. Although a firm must decrease its quantity drastically to satisfy $\sum_{i=1} q_{i} \leq 1-a$ (i.e., raising the market price by selling only to the consumers in $H$, see (1)), it is impossible to satisfy $\sum_{i=1} q_{i} \leq 1-a$ given $\sum_{j \neq i} q_{j}=(n-1)(1+b) /(n+1) \geq$ 1 under $b \geq 2 /(n-1)$. Therefore, no firm has an incentive to raise the market price even if the equilibrium market price is very low.

In general, the condition in Lemma 3 says the inverse. That is, firms sell only to high-end consumers if the low-end market is sufficiently unprofitable and/or inelastic.

It is noteworthy that this game has both equilibria under a certain range of $(a, b)$ because it is possible to satisfy both $a \geq(2-(n-1) b)^{2} /((n+1)(4-(n-3) b))$ and $a \leq 4 /((n+1)(4+(n+1) b))$.

\subsection{Comparison}

Using the results obtained so far, we determine the condition under which social welfare in case $I$ is smaller than that in case IIy.

First of all, $q^{y}$ must be a Cournot equilibrium in the absence of the public firm. ${ }^{13}$ From Lemma 2, $q^{y}$ becomes a Cournot equilibrium if one of the following inequalities is

\footnotetext{
${ }^{13}$ Otherwise, the supply of $h$ does not depend on whether or not the public firm exists in the low-end market. Therefore, the existence of the public firm is always preferable for social welfare.
} 
satisfied:

$$
a \geq \frac{(2-(n-1) b)^{2}}{(n+1)(4-(n-3) b)} \text { or } b \geq \frac{2}{n-1} .
$$

Second, we need $S W_{I}<S W_{I I y}$. That is, case $I$ is smaller than case IIy. This condition can be rewritten as follows:

$$
a<\frac{1}{2+b}
$$

Therefore, we obtain the following result.

Proposition 1 The nonexistence of the public firm can be beneficial from the viewpoint of social welfare if

$$
a^{B}(n)<a<\frac{1}{2+b}, \text { where } a^{B}(n) \equiv \begin{cases}\frac{(2-(n-1) b)^{2}}{(n+1)(4-(n-3) b)} & \text { if } b<\frac{2}{n-1}, \\ 0 & \text { if } b \geq \frac{2}{n-1}\end{cases}
$$

After some calculations, we obtain that both $2 /(n-1)$ and $(2-(n-1) b)^{2} /(n+1)(4-$ $(n-3) b)$ decrease with $n .{ }^{14}$ This implies that, as the number of private firms increases, the interval $\left[a^{B}(n), 1 /(2+b)\right]$ expands. Therefore, the existence of the public firm is more likely to worsen social welfare when there are many private firms in the high-end market.

Figure 2 shows the region in which this proposition holds $(n=2)$. It is noteworthy that $a<1 /(2+b)$ does not hold for any $b>0$ as long as $a>1 / 2$.

\section{[Figure 2 here]}

Note that, given that there are multiple equilibria (the value of $a$ is on the intermediate level mentioned above) in the absence of the public firm, the entry of the public does not always harm the social welfare.

The rough intuition of Proposition 1 is as follows. As case $I$ shows, the existence of a public firm makes private firms focus on the high-end market. This change brings about two effects: (i) the supply of the $l$ product becomes $b$ while (ii) the total supply of the $h$

\footnotetext{
14 The partial differentials of $(2-(n-1) b)^{2} /(n+1)(4-(n-3) b)$ and $2 /(n-1)$ with respect to $n$ are respectively $-8(1+b)^{2}(2-(n-1) b) /(n+1)^{2}(4-(n-3) b)^{2}$ and $-2 /(n-1)^{2}$. Both of them are negative.
} 
product becomes $n /(n+1)$ from $q^{y}$. Clearly, (i) is positive, and (ii) is negative from the viewpoint of social welfare.

However, if $q^{y}$ becomes an equilibrium in the absence of the public firm, we must take the negative effect of (ii) into account. There are some consumers in $H$ who would purchase the $h$ products if the public firm did not exist whether or not the welfare loss from such consumers in $H$ exceeds the welfare gain from consumers in $L$, who can obtain the $l$ product due to the entry of the public firm. The losses are imposed on a relatively small number of consumers in $H$, and the gains are imposed on a relatively large number of consumers in $L$. However, $p^{y}\left(q^{y}\right)<p^{x}\left(q^{x}\right)$ implies that the total willingness to pay of those consumers in $H$ is higher than that of the consumers in $L$. Therefore, the total effect depends on the demand structure that affects the impacts of (i) and (ii). The conditions in Proposition 1 are those under which (ii) outweighs (i).

\subsection{Privatization}

In the previous subsection, we compare the case in which a public firm exists with that in which no public firm exists.

In this subsection, we consider a case in which the public firm is privatized and $n$ private firms can potentially sell to both groups of consumers.

In this case, there are two candidates of Cournot equilibria:

$$
\begin{aligned}
& q^{z} \equiv\left(q_{0}^{z}, q_{1}^{z}, \ldots, q_{n}^{z}\right), \quad q^{w} \equiv\left(q_{0}^{w}, q_{1}^{w}, \ldots, q_{n}^{w}\right) \\
& \text { where } q_{0}^{z} \equiv \frac{b}{2}, q_{i}^{z} \equiv \frac{1}{n+1}(i=1, \ldots, n), q_{j}^{w} \equiv \frac{1+b}{n+2}(j=0,1, \ldots, n) .
\end{aligned}
$$

This is caused by our kinked inverse demand function. $q^{z}\left(\right.$ resp. $\left.q^{w}\right)$ is the Cournot equilibrium when $p^{h}>p^{l}\left(\right.$ resp. $\left.p^{h}=p^{l}\right)$ (see (1)). Therefore, we must check not only the local optimality (i.e., first-order condition) but also the global optimality (i.e., deviations "beyond" the kinked point). After several calculations, we obtain the following lemmas:

Lemma $4 q^{w}=((1+b) /(n+2), \ldots,(1+b) /(n+2))$ becomes a Cournot equilibrium if

$$
\frac{b(3-(n-1) b)^{2}}{(5-(n-3) b)((n+1) b-1)}<a, \quad \text { or } \quad b \geq \frac{3}{n-1} .
$$


The profit of each firm and social welfare are:

$$
\begin{aligned}
\pi_{I I I w} & \equiv \frac{a(1+b)^{2}}{(n+2)^{2}(a+b)}, \\
S W_{I I I w} & \equiv \frac{3 a+4 b-2 a b+4 a^{2} b+3 a b^{2}+(a+b)(1+a b) n(n+4)}{2(a+b)(n+2)^{2}} .
\end{aligned}
$$

Lemma $5 q^{z}=(b / 2,1 /(n+1), \ldots, 1 /(n+1))$ becomes a Cournot equilibrium if

$$
a \leq \frac{16}{(n+1)(8+(n+1) b)} .
$$

The profit of each private firm and social welfare are:

$$
\pi_{I I I z} \equiv \frac{1}{(n+1)^{2}}, \quad S W_{I I I z} \equiv \frac{3 a b+n(n+2)(4+3 a b)}{8(n+1)^{2}} .
$$

Lemma 4 says that $q^{w}$ becomes an equilibrium if the low-end market is sufficiently profitable (i.e., $a$ is relatively large for a given $b$ ) or large (i.e., $b$ is higher than $3 /(n-1)$ ). The condition in Lemma 5 says the inverse. That is, private firms sell only to high-end consumers if the low-end market is sufficiently unprofitable and/or inelastic. The values of $a$ are such that both conditions in Lemmas 4 and 5 are satisfied. On the other hand, if $b$ is small, neither $q^{w}$ nor $q^{z}$ can become an equilibrium.

If $q^{w}$ appears in equilibrium, privatization is better than the exit of the public firm from the viewpoint of social welfare because the total quantity supplied when the public firm is privatized is larger than that when the public firm exits. On the other hand, if $q^{z}$ appears in equilibrium, the existence of the public firm is better than privatization from the viewpoint of social welfare because privatization just decreases the quantity supplied by the former public firm.

From Lemma 4, we have the following proposition.

Proposition 2 Social welfare under privatization can be larger than that in which the public firm exists if

$$
\begin{aligned}
& a^{C}(n)<a<\frac{(n+2)^{2} b}{((n+1) b-1)((n+1) b+2 n+3)}, \\
& \text { where } a^{C}(n) \equiv \begin{cases}\frac{b(3-(n-1) b)^{2}}{(5-(n-3) b)((n+1) b-1)} & \text { if } b<\frac{3}{n-1}, \\
0 & \text { if } b \geq \frac{3}{n-1}\end{cases}
\end{aligned}
$$


Figures $3 \mathrm{a}$ and $3 \mathrm{~b}$ show the region in which this proposition holds $(n=2$ and $n$ is sufficiently large). It is noteworthy that $(n+2)^{2} b /(((n+1) b-1)((n+1) b+2 n+3))$ (line $(v i)$ ) converges to $1 /(2+b)$ (line $(i i i))$ as the value of $n$ increases.

[Figures 3a and 3b here]

When $n$ is small, the exit of the public firm tends to be superior to privatization. The privatized low-end firm lowers the price for low-end consumers, $p^{l}$, and then the lower price discourages the incentives of high-end firms to supply their products aggressively. Those high-end firms supply their products only for high-end consumers. The price for high-end consumers, $p^{h}$, becomes higher. When $n$ is large, however, the discouragement is not so significant because the larger number of high-end firms in itself enhances the total quantities supplied by those firms. The existence of the privatized firm additionally increases the total quantities supplied by those firms.

\section{Concluding remarks}

This paper discusses competition between high-quality private service providers that maximize their own profits and a low-quality public service provider that maximizes social surplus. There are two heterogeneous consumer groups: those who demand only highquality services and those who care little whether services are high- or low-quality. We show that, under certain conditions, the social welfare is smaller when there is a public service provider than when there is not. The result holds even though the efficiency of the public service is equal to that of the private service.

In this paper, following the literature of mixed oligopoly, we assume that the public firm tries to maximize social welfare and its production cost is as low as those of private firms. In reality, however, public firms might have other objectives, and they might suffer from an efficiency problem, such as moral hazard. Even in such situations, most of our arguments hold as long as a public firm tends to behave more aggressively in a market than private firms. In this sense, we can say that our arguments are robust with respect to those factors. 
In this paper, the quality of services provided by public firms is exogenously given. Investigating endogenous determinations of service qualities is an important matter. This is a relevant subject for future research.

$[2008.12 .1,903]$ 


\section{Appendix}

$n$ private firms with no public firm Before proofs of Lemmas 2 and 3, we first describe the setting of the model with $n$ private firms. We consider a case in which a public firm does not exist and the $n$ private firms can potentially sell to both groups of consumers. This is useful for proofs of Lemma 2 and 3.

We describe how the price $p^{h}$ is determined given the two groups of consumers. As long as $1-\sum_{i=1}^{n} q_{i} \geq a$, no consumer in $L$ buys $h$. Therefore, $p^{h}$ is given by

$$
p_{h}=1-\sum_{i=1}^{n} q_{i}
$$

If $1-\sum_{i=1}^{n} q_{i}<a$, some consumers in $L$ buy $h$. Because $h$ and $l$ are completely indifferent to consumers in $L$, this means that

$$
p^{h}=p^{l}=\frac{a\left(1+b-\sum_{i=1}^{n} q_{i}\right)}{a+b} .
$$

In summary, $p^{h}$ is determined as follows.

$$
p^{h}(q)= \begin{cases}1-\sum_{i=1}^{n} q_{i} & \text { if } \sum_{i=1}^{n} q_{i} \leq 1-a, \\ \frac{a\left(1+b-\sum_{i=1}^{n} q_{i}\right)}{a+b} & \text { otherwise. }\end{cases}
$$

Let $\pi_{i}(q)$ be the profit function of firm $i$. For $i=1,2, \ldots, n$, this can be expressed as follows.

$$
\pi_{i}(q)= \begin{cases}\left(1-\sum_{i=1}^{n} q_{i}\right) q_{i} & \text { if } \sum_{i=1}^{n} q_{i} \leq 1-a, \\ \frac{a\left(1+b-\sum_{i=1}^{n} q_{i}\right) q_{i}}{a+b} & \text { otherwise. }\end{cases}
$$

We solve the local optimal solutions in the two cases: (A) $\sum_{i=1}^{n} q_{i}$ is small, and (B) it is large. The first-order conditions lead to

$$
\begin{array}{r}
q_{i}^{A}(n)=\frac{1}{n+1}, \quad \sum_{i=1}^{n} q_{i}^{A}(n)=\frac{n}{n+1}, \quad \pi^{A}(n)=\frac{1}{(n+1)^{2}}, \quad \text { if } a \leq \frac{1}{n+1}, \\
\text { (B) } q_{i}^{B}(n)=\frac{1+b}{n+1}, \sum_{i=1}^{n} q_{i}^{B}(n)=\frac{n(1+b)}{n+1}, \quad \pi^{B}(n)=\frac{a(1+b)^{2}}{(a+b)(n+1)^{2}}, \\
\text { if } a \geq \max \left\{\frac{1-n b}{n+1}, 0\right\} .
\end{array}
$$


From (4), in each case, social welfare is described as:

$$
S W(n)= \begin{cases}\int_{0}^{\sum_{i=1}^{n} q_{i}}(1-x) d x=\frac{n^{2}}{2(n+1)^{2}}, & \text { case }(A), \\ \int_{0}^{1-a}(1-x) d x+\int_{1-a}^{\sum_{i=1}^{n} q_{i}} \frac{a(1+b-x)}{a+b} d x & \\ =\frac{(1-a)^{2} b+(a+b)(1+a b) n(n+2)}{2(a+b)(n+1)^{2}}, & \text { case }(B) .\end{cases}
$$

Proof of Lemma 2 We have to check whether the local optimal solution in (9) is also globally optimal.

In case (B), given the quantities supplied by the other firms, if a firm sets a smaller quantity $q_{d}^{B}(n)$, which satisfies $\sum_{i=1}^{n-1} q_{i}^{B}(n)+q_{d}^{B}(n) \leq 1-a$, the profit function is given by

$$
\pi_{d}^{B}(n)=\left(1-\frac{(n-1)(1+b)}{n+1}-q_{d}^{B}(n)\right) q_{d}^{B}(n) .
$$

When $b \geq 2 /(n-1)$, this is non-positive for any $q_{d}^{B}(n) \geq 0$, and then the interior solution in (9) is better for the deviating firm. When $b<2 /(n-1)$, the first-order condition leads to

$$
\begin{aligned}
& q_{d}^{B}(n)=\frac{2-(n-1) b}{2(n+1)}, \sum_{i=1}^{n-1} q_{i}^{B}(n)+q_{d}^{B}(n)=\frac{2 n+(n-1) b}{2(n+1)}, \\
& \pi_{d}^{B}(n)=\frac{(2-(n-1) b)^{2}}{4(n+1)^{2}}, \text { if } a \leq \frac{2-(n-1) b}{2(n+1)},
\end{aligned}
$$

otherwise, $q_{d}^{B}(n)$ is the corner solution, $q_{d}^{B}(n)=1-a-\sum_{i=1}^{n-1} q_{i}^{B}(n)$, and the interior solution in (9) is better for the deviating firm. Moreover, if $\pi_{d}^{B}(n)$ in (12) is smaller than or equal to $\pi^{B}$ in (9), the case (B) is an equilibrium outcome. The condition under which the solution in (9) is an equilibrium outcome is

$$
\frac{(2-(n-1) b)^{2}}{4(n+1)^{2}} \leq \frac{a(1+b)^{2}}{(a+b)(n+1)^{2}} \quad \text { or } \quad b \geq \frac{2}{n-1} .
$$

The condition can be summarized as follows:

$$
a \geq a^{B}(n)= \begin{cases}\frac{(2-(n-1) b)^{2}}{(n+1)(4-(n-3) b)} & \text { if } b<\frac{2}{n-1}, \\ 0 & \text { if } b \geq \frac{2}{n-1} .\end{cases}
$$

Therefore, Lemma 2 holds.

Q.E.D. 
Proof of Lemma 3 We have to check whether the local optimal solution in (8) is also globally optimal.

In case (A), given the quantities supplied by the other firms, if a deviating firm sets a larger quantity, $q_{d}^{A}(n)$, which satisfies $\sum_{i=1}^{n-1} q_{i}^{A}(n)+q_{d}^{A}(n) \geq 1-a$, the profit function is given by (see (7) and (8))

$$
\pi_{d}^{A}(n)=\frac{a\left(1+b-(n-1) /(n+1)-q_{d}^{A}(n)\right) q_{d}^{A}(n)}{a+b} .
$$

The first-order condition leads to

$$
\begin{aligned}
q_{d}^{A}(n)=\frac{2+(n+1) b}{2(n+1)}, & \sum_{i=1}^{n-1} q_{i}^{A}(n)+q_{d}^{A}(n)=\frac{2 n+(n+1) b}{2(n+1)}, \\
\pi_{d}^{A}(n)=\frac{a(2+(n+1) b)^{2}}{4(a+b)(n+1)^{2}}, & \text { if } a>a_{d}^{A}(n) \equiv \max \left\{\frac{2-(n+1) b}{2(n+1)}, 0\right\} ;
\end{aligned}
$$

otherwise, $q_{d}^{A}(n)$ is the corner solution, $q_{d}^{A}(n)=1-a-\sum_{i=1}^{n-1} q_{i}^{A}(n)$, and the interior solution in (8) is better for the deviating firm. That is, if $a<a_{d}^{A}(n)$, the interior solution in (8) is an equilibrium outcome. Moreover, if $\pi_{d}^{A}(n)$ in (15) is smaller than or equal to $\pi^{A}(n)$ in (8), the quantities in case (A) are optimal. The condition under which the solution in (8) is an equilibrium outcome is

$$
\frac{a(2+(n+1) b)^{2}}{4(a+b)(n+1)^{2}} \leq \frac{1}{(n+1)^{2}} \text { or } a \leq \max \left\{\frac{2-(n+1) b}{2(n+1)}, 0\right\} .
$$

The latter condition is redundant. The condition under which case (A) is an equilibrium outcome is

$$
a \leq a^{A}(n) \equiv \frac{4}{(n+1)(4+(n+1) b)} .
$$

Therefore, Lemma 3 holds.

Q.E.D.

Proof of Lemma 4 We have to check whether the local optimal solution in Lemma 4 is also globally optimal.

Given the quantities supplied by the other firms, if a firm sets a smaller quantity $q_{d}^{w}$, which satisfies $\sum_{i=1}^{n-1} q_{i}^{w}+q_{d}^{w} \leq 1-a+a q_{0}^{w} / b$, the profit function is given by

$$
\pi_{d}^{w}=\left(1-\frac{(n-1)(1+b)}{n+2}-q_{d}^{w}\right) q_{d}^{w}
$$


When $b \geq 3 /(n-1)$, this is non-positive for any $q_{d}^{w} \geq 0$, and then the interior solution in Lemma 4 is better for the deviating firm. When $b<3 /(n-1)$, the first-order condition leads to

$$
\begin{aligned}
q_{d}^{w}=\frac{3-(n-1) b}{2(n+2)}, \sum_{i=1}^{n-1} q_{i}^{w}+q_{d}^{w}= & \frac{2 n+1+(n-1) b}{2(n+2)}, \\
& \pi_{d}^{w}=\frac{(3-(n-1) b)^{2}}{4(n+2)^{2}}, \text { if } a \leq \frac{b(3-(n-1) b)}{2(b(n+1)-1)}
\end{aligned}
$$

otherwise, $q_{d}^{w}$ is the corner solution, $q_{d}^{w}=1-a-\sum_{i=1}^{n-1} q_{i}^{w}+a q_{0}^{w} / b$, and the interior solution in Lemma 4 is better for the deviating firm. Moreover, if $\pi_{d}^{w}$ in (18) is smaller than or equal to $\pi_{I I I w}$ in Lemma 4 , this is an equilibrium outcome. The condition under which the solution is an equilibrium outcome is

$$
\frac{(3-(n-1) b)^{2}}{4(n+2)^{2}} \leq \frac{a(1+b)^{2}}{(a+b)(n+2)^{2}} \quad \text { or } \quad b \geq \frac{3}{n-1} .
$$

The condition can be summarized as follows:

$$
a \geq a^{C}(n)= \begin{cases}\frac{b(3-(n-1) b)^{2}}{(5-(n-3) b)((n+1) b-1)} & \text { if } b<\frac{3}{n-1}, \\ 0 & \text { if } b \geq \frac{3}{n-1} .\end{cases}
$$

Therefore, Lemma 4 holds.

Q.E.D.

Proof of Lemma 5 We have to check whether the local optimal solution is also globally optimal.

Given the quantities supplied by the other firms, if a deviating firm sets a larger quantity, $q_{d}^{z}$, which satisfies $\sum_{i=1}^{n-1} q_{i}^{z}+q_{d}^{z} \geq 1-a+a q_{0}^{z} / b$, the profit function is given by

$$
\pi_{d}^{z}=\frac{a\left(1+b-(n-1) /(n+1)-b / 2-q_{d}^{z}\right) q_{d}^{z}}{a+b}
$$

The first-order condition leads to

$$
\begin{aligned}
q_{d}^{z}=\frac{4+(n+1) b}{4(n+1)}, & \sum_{i=1}^{n-1} q_{i}^{z}+q_{d}^{z}=\frac{4 n+(n+1) b}{4(n+1)} \\
& \pi_{d}^{z}=\frac{a(4+(n+1) b)^{2}}{16(a+b)(n+1)^{2}}, \quad \text { if } a>a_{d}^{z} \equiv \max \left\{\frac{4-(n+1) b}{2(n+1)}, 0\right\} ;
\end{aligned}
$$


otherwise, $q_{d}^{z}$ is the corner solution, $q_{d}^{z}=1-a / 2-\sum_{i=1}^{n-1} q_{i}^{z}$, and the interior solution is better for the deviating firm. That is, if $a<a_{d}^{z}$, the interior solution is an equilibrium outcome. Moreover, if $\pi_{d}^{A}(n)$ in (21) is smaller than or equal to $\pi_{I I I z}$ in Lemma 5 , the quantities $q^{z}$ are optimal. The condition under which the solution in Lemma 5 is an equilibrium outcome is

$$
\frac{a(4+(n+1) b)^{2}}{16(a+b)(n+1)^{2}} \leq \frac{1}{(n+1)^{2}} \text { or } a \leq \max \left\{\frac{2-(n+1) b}{2(n+1)}, 0\right\} .
$$

The latter condition is redundant. The condition under which this is an equilibrium outcome is

$$
a \leq \frac{16}{(n+1)(8+(n+1) b)}
$$

Therefore, Lemma 5 holds.

Q.E.D. 


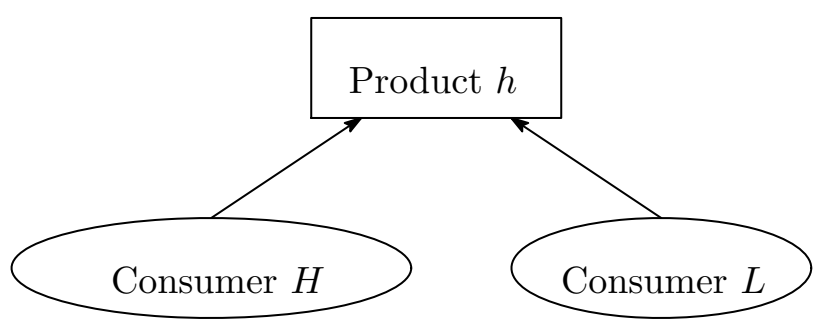

[No public firm exists]

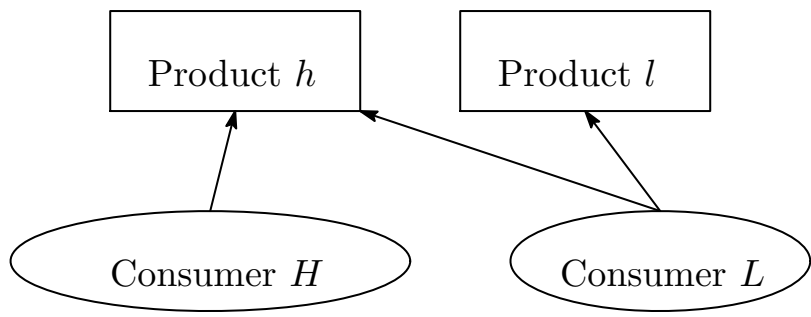

[A public firm exists]

Figure 1: The market structure 


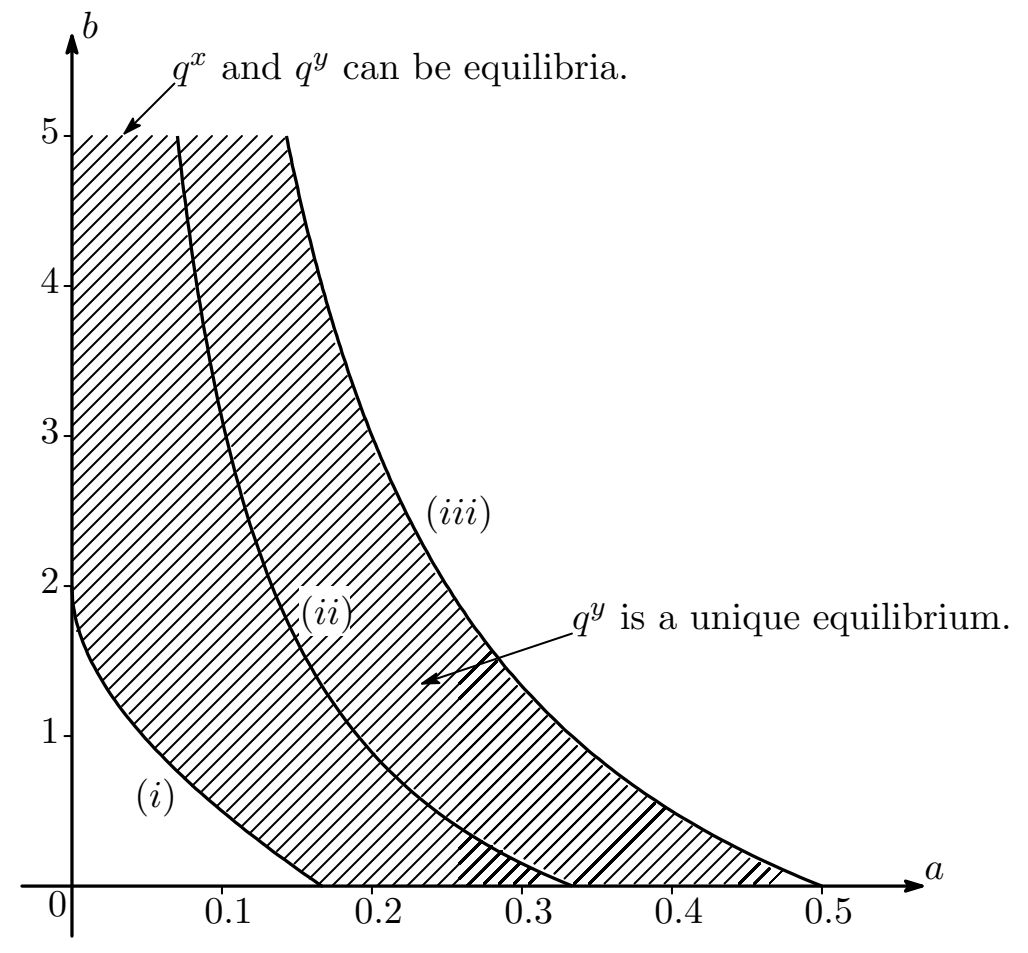

Figure 2: Parameter range within which the public firm can be harmful.

(Horizontal: $a$, Vertical: $b$ )

Note: $\quad(i) a=\frac{(2-(n-1) b)^{2}}{(n+1)(4-(n-3) b)}, \quad($ ii $) a=\frac{4}{(n+1)(4+(n+1) b)}, \quad$ (iii) $a=\frac{1}{2+b}$. 


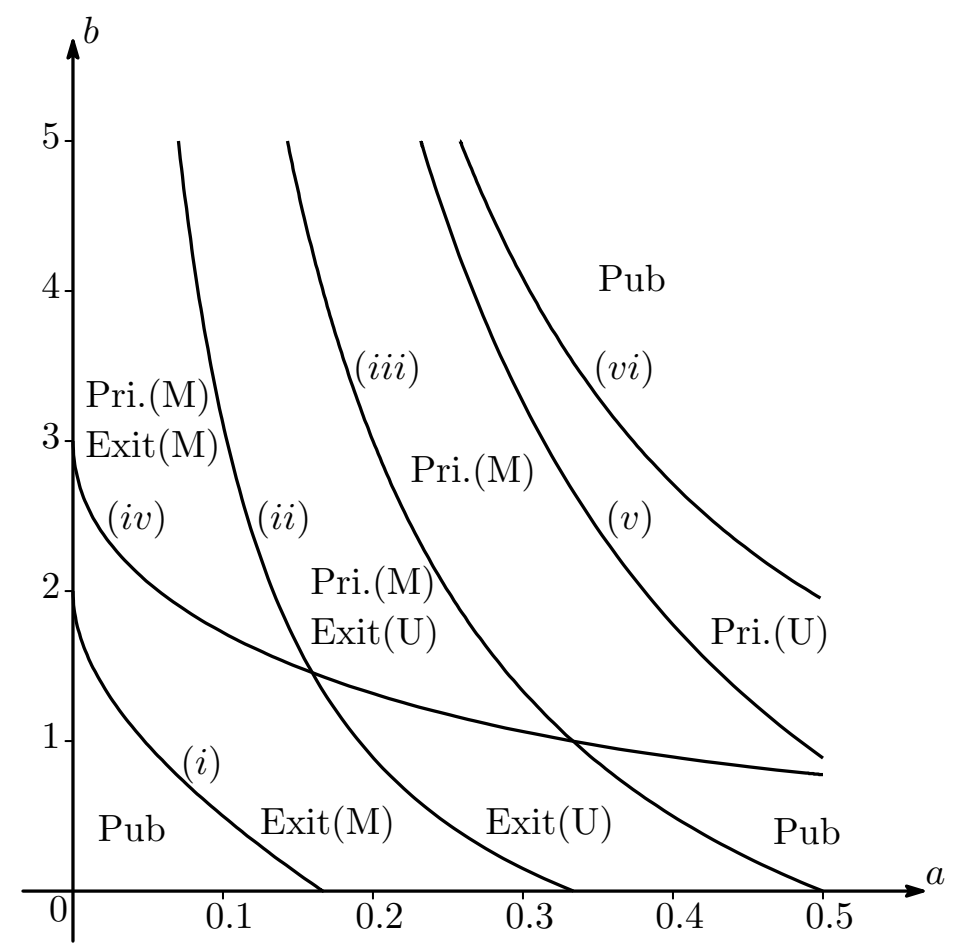

Figure 3a: A comparison of the three cases $(n=2)$.

(Horizontal: $a$, Vertical: $b$ )

Note 1: $\quad(i) a=\frac{(2-(n-1) b)^{2}}{(n+1)(4-(n-3) b)}, \quad($ ii $) a=\frac{4}{(n+1)(4+(n+1) b)}, \quad$ (iii) $a=\frac{1}{2+b}$,

(iv) $a=\frac{b(3-(n-1) b)^{2}}{(5-(n-3) b)((n+1) b-1)}, \quad(v) a=\frac{16}{(n+1)(8+(n+1) b)}$,

(vi) $a=\frac{(n+2)^{2} b}{((n+1) b-1)(3+2 n+(n+1) b)}$.

Note 2:

Pri.(U): Privatization is superior to the existence of the public firm. ( $q^{w}$ appears in equilibrium (a unique equilibrium).)

Pri.(M): Privatization can be superior to the existence of the public firm. (Both $q^{w}$ and $q^{z}$ can appear in equilibrium (multiple equilibria).)

Exit(U): The exit of the public firm is superior to its existence. ( $q^{y}$ appears in equilibrium (a unique equilibrium).)

Exit(M): The exit of the public firm can be superior to its existence. (Both $q^{x}$ and $q^{y}$ can appear in equilibrium (multiple equilibria).)

Pub.: $\quad$ The existence of the public firm is superior to the rest of the cases. 


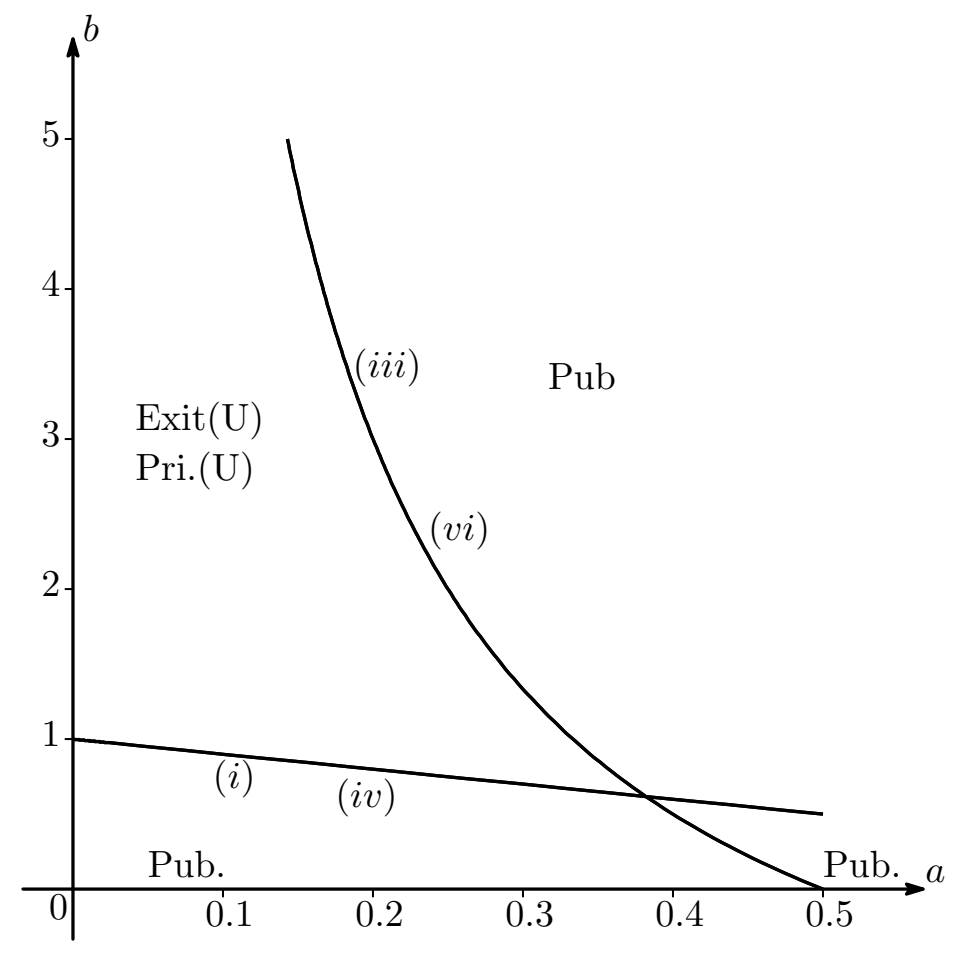

Figure 3b: A comparison of the three cases ( $n$ is sufficiently large).

(Horizontal: $a$, Vertical: $b$ )

Note 1: $\quad(i) a=\frac{(2-(n-1) b)^{2}}{(n+1)(4-(n-3) b)}, \quad$ (ii) $a=\frac{4}{(n+1)(4+(n+1) b)}, \quad$ (iii) $a=\frac{1}{2+b}$,

$$
\begin{gathered}
\text { (iv) } a=\frac{b(3-(n-1) b)^{2}}{(5-(n-3) b)((n+1) b-1)}, \quad(v) a=\frac{16}{(n+1)(8+(n+1) b)}, \\
(v i) a=\frac{(n+2)^{2} b}{((n+1) b-1)(3+2 n+(n+1) b)} .
\end{gathered}
$$

Note 2:

Pri.(U): Privatization is superior to the existence of the public firm.

( $q^{w}$ appears in equilibrium (a unique equilibrium).)

Exit(U): The exit of the public firm is superior to its existence. ( $q^{y}$ appears in equilibrium (a unique equilibrium).)

Pub.: The existence of the public firm is superior to the rest of the cases. 


\section{References}

Akin, John and Paul Hutchinson. 1999. "Health-care Facility Choice and the Phenomenon of Bypassing." Health Policy and Planning 14: 135-51.

Anderson, Simon P., Andre de Palma, and Jacques-François Thisse. 1997. "Privatization and Efficiency in a Differentiated Industry." European Economic Review 41: 16351654 .

Barros, Pedro Pita and Xavier Martinez-Giralt. 2002. "Public and Private Provision of Health Care." Journal of Economics and Management Strategy 11: 109-133.

Barros, Pedro Pita and Pau Olivella. 2005. "Waiting Lists and Patient Selection." Journal of Economics and Management Strategy 14: 623-646.

Biglaiser, Gary and Ching-to Albert Ma. 2007. "Moonlighting: Public Service and Private Practice." RAND Journal of Economics 38: 1113-1133.

Bös, Dieter. 1986. Public Enterprise Economics, North-Holland, Amsterdam.

Bös, Dieter. 1991. Privatization: A Theoretical Treatment, Clarendon Press, Oxford.

Brekke, Kurt R. and Lars Sørgard. 2007. "Public versus private health care in a national health service." Health Economics 16: 579-601.

Cremer, Helmuth, Maurice Marchand and Jacques-François Thisse. 1991. "Mixed Oligopoly with Differentiated Products." International Journal of Industrial Organization 9: 43-53.

De Fraja, Gianni and Flavio Delbono. 1989. "Alternative Strategies of a Public Enterprise in Oligopoly." Oxford Economic Papers 41: 302-311.

García-Prado, Ariadna and Paula Gonzàlez. 2007. "Policy and Regulatory Responses to Dual Practice in the Health Sector." Health Policy 84: 142-152.

Gauthier, Bernard and Waly Wane. 2008. "Bypassing Health Providers: The Quest for Better Price and Quality of Health Care in Chad." Policy Research Working Paper Series 4462, The World Bank.

Glazer, Amihai and Esko Niskanen. 1997. "Why voters may prefer congested public clubs." Journal of Public Economics 65: 37-44.

Gonzàlez, Paula. 2004. "Should physicians' dual practice be limited? An incentive approach." Health Economics 13: 505-524.

Ishibashi, Ikuo and Toshihiro Matsumura. 2006. "R\&D Competition between public and private sectors." European Economic Review 50: 1347-1366.

Ishibashi, Ikuo and Noriaki Matsushima. 2008. "The existence of low-end firms may help high-end firms." Marketing Science forthcoming. 
Leonard, Kenneth L., Gilbert R. Mliga, and Damen Haile Mariam. 2002. "Bypassing Health Centres in Tanzania: Revealed Preferences for Quality," Journal of African Economies 11: 441-471.

Li, Changying. 2006. "Location choice in a mixed oligopoly." Economic Modelling 23: $131-41$.

Lu, Yuanzhu. 2006. "Endogenous timing in a mixed oligopoly with foreign competitors: the linear demand case." Journal of Economics 88: 49-68.

Lu, Yuanzhu and Sougata Poddar. 2007. "Firm ownership, product differentiation and welfare." Manchester School 75: 210-217.

Ma, Ching-to Albert. 2003. "Public Rationing and Private Cost Incentives." Journal of Public Economics 88: 333-352.

March, Maurice and Fred Schroyen. 2005. "Can a Mixed Health Care System be Desirable on Equity Grounds?" Scandinavian Journal of Economics 107: 1-23.

Matsumura, Toshihiro. 1998. "Partial Privatization in Mixed Duopoly." Journal of Public Economics 70: 473-483.

Matsumura, Toshihiro. 2003. "Stackelberg mixed duopoly with a foreign competitor." Bulletin of Economic Research 55: 275-287.

Matsumura, Toshihiro and Noriaki Matsushima. 2004. "Endogenous Cost Differentials between Public and Private Enterprises: A Mixed Duopoly Approach." Economica 71: $671-688$.

Matsushima, Noriaki and Toshihiro Matsumura. 2003. "Mixed Oligopoly and Spatial Agglomeration." Canadian Journal of Economics 36: 62-87.

Matsushima, Noriaki and Toshihiro Matsumura. 2006. "Mixed oligopoly, foreign firms, and location choice." Regional Science and Urban Economics 36: 753-772.

Merrill, William and Norman Schneider. 1966. "Government Firms in Oligopoly Industries: A Short-run Analysis." Quarterly Journal of Economics 80: 400-412.

Nett, Lorenz. 1993. "Mixed Oligopoly with Homogeneous Goods." Annals of Public and Cooperative Economics 64: 367-93.

Pal, Debashis. 1998. "Endogenous timing in a mixed oligopoly." Economics Letters 61: $181-85$.

Poyago-Theotoky, Joanna A. 1998. "R\&D Competition in a Mixed Duopoly under Uncertainty and Easy Imitation." Journal of Comparative Economics 26: 415-428.

Rickman, Neil and Alistair McGuire. 1999. "Regulating Providers' Reimbursement in a Mixed Market for Health Care." Scottish Journal of Political Economy 46: 53-71. 